\title{
INTERFERENCE OF BREAST CANCER COMMUNICATION ON TREATMENT ADHERENCE AND THE DOCTOR-PATIENT RELATIONSHIP
}

Pedro Henrique de Ávila Perillo1, Vinicius Lemos Nascimento1', Laís Martins Queiroz¹, Ianca Leandra Santos', Ana Carla Moreira Mirandaㅁ, Débora Sara de Almeida Cardoso ${ }^{1}$

${ }^{1}$ Faculdade de Medicina, Hospital das Clínicas, Universidade Federal de Goiás - Goiânia (GO), Brazil.

Objectives: Breast cancer is the cancer with the highest incidence in women in Brazil. The revelation of this diagnosis is considered a crucial moment, responsible for uncertainties and even affecting the doctor-patient relationship. Although the communication of bad news is one of the most frequent practices among physicians, difficulties are common. The aim of this study was to evaluate the influence of the communication in the diagnosis of breast cancer and the repercussions on the treatment, using the parameters as suggested by the Centro Avançado de Diagnóstico de Câncer de Mama (CORA/ HC-UFG) in Goiânia-GO. Methodology: This qualitative and integrative study was conducted in the following databases: PubMed, SciELO, and MedLine with six selected articles. The keywords used were "communication," "diagnosis," "breast cancer," "adherence to treatment," and "doctor-patient relationship." Based on the results, a comparison was made with the one carried out in the CORA/HC-UFG. Results: Through the analysis of the articles, it was observed that the no tification of the diagnosis of cancer is considered a critical moment and how professionals notify patients directly interferes in the relationship of the patients with the diagnosis itself. Thus, it is up to the professionals to provide psychological and informative support at the time of diagnosis in order to reduce pessimistic feelings and to avoid abandoning the medical-hospital follow-up. In addition, when analyzing the care provided at CORA, it was noted that the adoption of a more humanized and multiprofessional communication, with the help of the psychological team, it was noticed, in agreement with the studies, a better acceptance and treatment adherence. Conclusion: The notification of cancer should be done in the most empathetic way possible, using techniques of psychology and communication, so that the patient receives information about his health situation in a welcoming manner. Furthermore, although the diagnostic communication is a medical act, the presence of a psychologist to support the patient has shown benefits.

Keywords: Communication; Diagnosis; Breast Cancer; Treatment Adherence; Doctor-Patient Relationship. 